\title{
Short communication: Supplementation of fructo-oligosaccharides does not improve insulin sensitivity in heavy veal calves fed different sources of carbohydrates
}

\author{
A. J. Pantophlet, ${ }^{* 1}$ M. S. Gilbert, † W. J. J. Gerrits, $†$ and R. J. Vonkł \\ *Department of Pediatrics, Center for Liver, Digestive and Metabolic Diseases, University Medical Centre Groningen, PO Box 30001 , \\ 9700 RB Groningen, the Netherlands \\ †Animal Nutrition Group, Wageningen University, PO Box 338, $6700 \mathrm{AH}$, Wageningen, the Netherlands \\ ¥Centre for Medical Biomics, University Medical Center Groningen, PO Box 30001, 9700 RB, Groningen, the Netherlands
}

\begin{abstract}
Heavy veal calves (4-6 mo old) often develop problems with insulin sensitivity. This could lead to metabolic disorders and impaired animal growth performance. Studies in various animal species have shown that the supplementation of short-chain fructo-oligosaccharides (scFOS) can improve insulin sensitivity. We therefore studied the effects of scFOS supplementation on insulin sensitivity in heavy veal calves. Forty male HolsteinFriesian calves $(\mathrm{BW}=190 \pm 2.9 \mathrm{~kg}$; age $=162 \pm 1.4 \mathrm{~d}$ at the start of the trial) were fed either a control milk replacer (MR) diet or a diet in which one-third of the lactose was replaced by glucose, fructose, or glycerol for $10 \mathrm{wk}$ prior to the start of the trial. At the start of the trial, calves were subjected to a frequently sampled intravenous glucose tolerance test to assess whole-body insulin sensitivity (muscle and hepatic insulin sensitivity). Calves within each dietary treatment group were ranked based on their insulin sensitivity value. Half of the calves received scFOS $(12 \mathrm{mg} / \mathrm{kg}$ of BW) with the MR for $6 \mathrm{wk}$ (supplementation was equally distributed over the insulin sensitivity range). Subsequently, a second frequently sampled intravenous glucose tolerance test was conducted to assess the effect of scFOS. In addition, fasting plasma levels of glucose, insulin, triglycerides, and cholesterol were determined to calculate the quantitative insulin sensitivity check index and triglyceride:high-density lipoprotein cholesterol ratio (fasting indicators of insulin sensitivity). Whole-body insulin sensitivity was low at the start of the trial and remained low in all groups $[1.0 \pm 0.1$ and $0.8 \pm 0.1$ $(\mathrm{mU} / \mathrm{L})^{-1} \cdot \min ^{-1}$ on average, respectively]. Supplementation of scFOS did not improve insulin sensitivity in any of the treatment groups. The quantitative insulin
\end{abstract}

Received March 7, 2017.

Accepted July 2, 2017.

${ }^{1}$ Corresponding author: a.j.pantophlet@umcg.nl sensitivity check index and the triglyceride:high-density lipoprotein cholesterol ratio also did not differ between scFOS and non-scFOS calves and averaged $0.326 \pm$ 0.003 and $0.088 \pm 0.004$, respectively, at the end of the trial. We conclude that scFOS supplementation does not improve insulin sensitivity in heavy veal calves regardless of the carbohydrate composition of the MR. This is in contrast to other animals (e.g., dogs and horses), where scFOS supplementation did improve insulin sensitivity. The absence of an effect of scFOS might be related to the dosage or to metabolic differences between ruminants and nonruminants. Increasing evidence indicates that dietary interventions in veal calves have little or no effect on insulin sensitivity, possibly because of low levels of insulin sensitivity.

Key words: veal calf, milk replacer, fructooligosaccharides, insulin sensitivity

\section{Short Communication}

Veal calves are fed milk replacer (MR) and solid feed (roughage and concentrates) and are exposed to large quantities of lactose and fat via the MR. Prolonged high intakes of MR can induce problems with glucose homeostasis and insulin sensitivity in veal calves. These problems have been identified in heavy veal calves (4-6 mo), characterized by a high incidence of hyperglycemia, hyperinsulinemia, and glucosuria (Hostettler-Allen et al., 1994; Hugi et al., 1997) and insulin resistance (Pantophlet et al., 2016a). In a recent study, we found that calves raised on a lactose MR diet, or diets in which one-third of the lactose was replaced by glucose, fructose, or glycerol, did not differ in insulin sensitivity and that insulin sensitivity was low (Pantophlet et al., 2016c). This could lead to metabolic disorders and impaired animal growth performance. Therefore, prevention strategies must be developed. Various animal studies have shown that dietary short-chain fructo-oligosaccharide (scFOS) supplementation can 
help prevent problems and improve whole-body insulin sensitivity (i.e., muscle and hepatic insulin sensitivity). In obese dogs and horses, for example, supplementation of dietary scFOS for a period of $6 \mathrm{wk}$ increased insulin sensitivity (Respondek et al., 2008; Respondek et al., 2011). In young veal calves ( $<3$ mo old), supplementation of scFOS for 10 wk did not improve insulin sensitivity or glucose homeostasis (Pantophlet et al., 2016b). In older veal calves (10-13 wk old), however, scFOS supplementation did improve postprandial glucose homeostasis (Kaufhold et al., 2000). A decrease in postprandial response for glucose was observed, but not for insulin. Mechanisms were not reported. It is not clear whether the improved glucose homeostasis during scFOS supplementation in older calves is related to changes in insulin sensitivity. Therefore, the objective of this study was to assess the effects of scFOS supplementation on insulin sensitivity in heavy veal calves.

Forty male Holstein-Friesian calves $(\mathrm{BW}=190 \pm$ $2.9 \mathrm{~kg}$; age $=162 \pm 1.4 \mathrm{~d}$; mean $\pm \mathrm{SEM}$ ) were housed at the research facility of the Department of Animal Sciences at Wageningen University (Wageningen, the Netherlands). Calves were housed in groups (5 calves/ pen) except for the first and last week of the trial. During these periods calves were housed individually in metabolic cages $(0.80 \times 1.8 \mathrm{~m})$. Experimental procedures complied with the Dutch Law on Experimental Animals and the ETS123 (Council of Europe 1985 and the 86/609/EEC Directive) and were approved by the Animal Care and Use Committee of Wageningen University. Prior to the start of the trial, the calves were fed either a control MR diet $(n=10)$ or diets in which one-third of the lactose in the MR was replaced by isoenergetic amounts of glucose (Tereos Syral, Marckolsheim, France; $\mathrm{n}=10$ ), fructose (Tate and Lyle Europe, Boleraz, Slovakia; $n=10$ ), or glycerol (Triconor Distribution BV, Soest, the Netherlands; $\mathrm{n}=10$ ) for $10 \mathrm{wk}$. All calves remained on their diets throughout the trial. A detailed description of the diets and feeding strategy is provided elsewhere (Pantophlet et al., 2016c). In short, calves were fed MR and solid feed (20\% wheat straw and $80 \%$ concentrates) twice a day, at 0630 and $1530 \mathrm{~h}$. The MR was fed on an individual basis (BW was measured weekly), and solid feed was provided per pen during group housing and per individual calf when the calves were housed in metabolic cages. Calves had ad libitum access to water. Whole-body insulin sensitivity was assessed at the start and the end of the trial. The start values were used to assign calves to scFOS or no scFOS within each dietary treatment group (i.e., n $=5$ for $\mathrm{scFOS} ; \mathrm{n}=5$ for no $\mathrm{scFOS}$ ). The calves were ranked to evenly distribute the supplementation scFOS over the insulin sensitivity range in each group. The
scFOS were added to the MR at a dose of $12 \mathrm{mg} / \mathrm{kg}$ of BW for $6 \mathrm{wk}$. At the end of the 6 -wk period, wholebody insulin sensitivity was assessed again to study the effect of scFOS supplementation. One calf in the glucose group was excluded from all measurements due to ruminal drinking.

Whole-body insulin sensitivity was assessed using the frequently sampled intravenous glucose tolerance test. A detailed description of this experimental procedure is provided elsewhere (Pantophlet et al., 2016c). In short, a central venous catheter (Careflow, Becton Dickinson, Franklin Lakes, NJ) was inserted in the jugular vein for glucose and insulin infusion and blood sampling. Calves were fasted to achieve a steady glucose turnover rate before the test. At $t=0 \mathrm{~min}$, an intravenous glucose bolus of $0.3 \mathrm{~g} / \mathrm{kg}$ of BW (20\% glucose solution; B. Braun, Oss, the Netherlands) was administered within 2 min followed by an intravenous insulin bolus of $0.03 \mathrm{IU} / \mathrm{kg}$ of BW (100 IU/mL solution; Insuman Rapid, Sanofi-Aventis, Gouda, the Netherlands) at $t$ $=20 \mathrm{~min}$ (administered within $1 \mathrm{~min}$ ). Blood samples were collected at $t=-8,-4,2,4,6,8,10,12,14,16$, $19,22,25,30,35,40,50,60,75,90,120,150$, and 180 min relative to the glucose bolus. The samples were centrifuged $(1,516 \times g$ for $10 \mathrm{~min})$, and plasma was harvested for the analysis of plasma glucose and insulin concentrations. In addition, plasma triglycerides and high-density lipoprotein (HDL) cholesterol concentrations were analyzed in the fasting plasma sample collected at $t=-8 \mathrm{~min}$ (at the start and the end of the trial). Whole-body insulin sensitivity was calculated according to Bergman's minimal model approach using MinMod Millennium (version 6.0.2; MinMod Inc., Los Angeles, CA). In addition, another index of insulin sensitivity, the quantitative insulin sensitivity index (QUICKI; Muniyappa et al., 2008), was calculated from the fasting plasma glucose and insulin concentrations. Also, clean urine was quantitatively collected for a period of 5 and $3 \mathrm{~d}$ at the start and the end of the trial, respectively. A detailed description of the urine collection procedure is provided elsewhere (Pantophlet et al., 2016c). Urinary glucose and plasma glucose, triglycerides, and HDL cholesterol were measured on a Roche-Hitachi modular automatic analyzer (Roche Diagnostics, Basel, Switzerland) using enzymatic colorimetric assays. The within- and between-run coefficients of variation were $\leq 2 \%$ for all analyses. Insulin was measured using a bovine ELISA kit (Mercodia, Uppsala, Sweden). The within- and between-run coefficients of variation were $\leq 5.6$ and $8.2 \%$, respectively.

Data were analyzed using SPSS (version 22; IBM, Armonk, NY). The effect of scFOS on whole-body insulin sensitivity, QUICKI, triglyceride:HDL cholesterol 
ratio, fasting plasma glucose and insulin, and urinary glucose excretion was tested by ANOVA using the GLM (Univariate) procedure. The overall effect of scFOS and the within-treatment effect (i.e., within the several diets) were tested. Supplementation with scFOS (i.e., scFOS vs. non-scFOS calves) was used as a factor, and calf was the experimental unit. The final values (i.e., measured at the end of the trial) were used as dependent variables, with their respective initial values as covariates. For the overall effects an scFOS $x$ dietary treatment interaction was included. $P$-values $<$ 0.05 were considered significant.
The average daily BW gain, measured over the whole trial period, was on average $1.19 \pm 0.02 \mathrm{~kg} / \mathrm{d}$ and did not differ between dietary treatments or scFOS treatment. Feed refusal was negligible throughout the trial. Whole-body insulin sensitivity calculated using MinMod Millennium was low at the start of the trial (it did not differ between scFOS and non-scFOS calves) and remained low until the end $[1.0 \pm 0.1$ and $0.8 \pm 0.1$ $(\mathrm{mU} / \mathrm{L})^{-1} \cdot \min ^{-1}$ on average at the start and the end of the trial, respectively]. Supplementation of scFOS did not improve insulin sensitivity $(P=0.324$; Table 1). During the trial, fasting plasma glucose levels de-

Table 1. Insulin sensitivity, triglyceride:high-density lipoprotein (HDL) cholesterol ratio, fasting glucose, and insulin and urinary glucose excretion (mean \pm SEM) at the end of the trial in veal calves fed a control milk replacer $(\mathrm{n}=10)$ or a milk replacer in which one-third of the lactose was replaced by isoenergetic amounts of glucose $(n=9)$, fructose $(n=10)$, or glycerol $(n=10)$

\begin{tabular}{|c|c|c|c|c|}
\hline \multirow[b]{2}{*}{ Item $^{1}$} & \multicolumn{2}{|c|}{ Treatment $^{2}$} & \multirow[b]{2}{*}{$P$-value } & \multirow{2}{*}{$\begin{array}{c}\text { Interaction } \\
P \text {-value }\end{array}$} \\
\hline & scFOS & No scFOS & & \\
\hline \multicolumn{5}{|c|}{$\overline{\mathrm{IS}_{\text {minmod }} \times 10^{-4},(\mathrm{mU} / \mathrm{L})^{-1} \min ^{-1}}$} \\
\hline Overall & $0.79 \pm 0.14$ & $0.89 \pm 0.13$ & 0.324 & \multirow[t]{5}{*}{0.463} \\
\hline Control & $0.74 \pm 0.14$ & $1.01 \pm 0.34$ & 0.291 & \\
\hline Glucose & $0.71 \pm 0.27$ & $0.83 \pm 0.10$ & 0.852 & \\
\hline Fructose & $1.36 \pm 0.39$ & $1.00 \pm 0.27$ & 0.505 & \\
\hline Glycerol & $0.44 \pm 0.10$ & $0.62 \pm 0.10$ & 0.130 & \\
\hline \multicolumn{5}{|l|}{ QUICKI } \\
\hline Overall & $0.324 \pm 0.004$ & $0.329 \pm 0.005$ & 0.434 & \multirow[t]{5}{*}{0.734} \\
\hline Control & $0.308 \pm 0.011$ & $0.323 \pm 0.011$ & 0.558 & \\
\hline Glucose & $0.320 \pm 0.005$ & $0.342 \pm 0.007$ & 0.063 & \\
\hline Fructose & $0.340 \pm 0.013$ & $0.325 \pm 0.003$ & 0.807 & \\
\hline Glycerol & $0.324 \pm 0.006$ & $0.331 \pm 0.007$ & 0.639 & \\
\hline \multicolumn{5}{|c|}{ Triglyceride:HDL cholesterol ratio } \\
\hline Overall & $0.087 \pm 0.006$ & $0.090 \pm 0.007$ & 0.191 & \multirow[t]{5}{*}{0.288} \\
\hline Control & $0.089 \pm 0.012$ & $0.106 \pm 0.012$ & 0.090 & \\
\hline Glucose & $0.095 \pm 0.009$ & $0.103 \pm 0.026$ & 0.160 & \\
\hline Fructose & $0.067 \pm 0.016$ & $0.079 \pm 0.003$ & 0.482 & \\
\hline Glycerol & $0.091 \pm 0.013$ & $0.077 \pm 0.009$ & 0.430 & \\
\hline \multicolumn{5}{|c|}{ Fasting glucose, $\mathrm{mmol} / \mathrm{L}$} \\
\hline Overall & $5.1 \pm 0.1$ & $5.0 \pm 0.1$ & 0.100 & \multirow[t]{5}{*}{0.069} \\
\hline Control & $5.0 \pm 0.2$ & $5.1 \pm 0.2$ & 0.574 & \\
\hline Glucose & $4.8 \pm 0.2$ & $5.1 \pm 0.1$ & 0.295 & \\
\hline Fructose & $5.3 \pm 0.1$ & $5.1 \pm 0.2$ & 0.295 & \\
\hline Glycerol & $5.4 \pm 0.1$ & $5.1 \pm 0.1$ & 0.171 & \\
\hline \multicolumn{5}{|c|}{ Fasting insulin, $\mathrm{mU} / \mathrm{L}$} \\
\hline Overall & $15.5 \pm 2.2$ & $12.7 \pm 1.3$ & 0.204 & \multirow{5}{*}{0.397} \\
\hline Control & $23.2 \pm 9.4$ & $16.1 \pm 3.8$ & 0.467 & \\
\hline Glucose & $16.0 \pm 1.7$ & $9.6 \pm 1.9$ & 0.062 & \\
\hline Fructose & $10.6 \pm 1.7$ & $13.1 \pm 1.0$ & 0.629 & \\
\hline Glycerol & $15.5 \pm 2.2$ & $12.7 \pm 1.3$ & 0.615 & \\
\hline \multicolumn{5}{|c|}{ Urinary glucose excretion, $\mathrm{g} / \mathrm{d}$} \\
\hline Overall & $48.5 \pm 14.2$ & $31.8 \pm 8.6$ & 0.243 & \multirow{5}{*}{0.435} \\
\hline Control & $49.5 \pm 21.7$ & $37.3 \pm 10.9$ & 0.438 & \\
\hline Glucose & $126.9 \pm 20.3$ & $83.4 \pm 15.8$ & 0.298 & \\
\hline Fructose & $7.0 \pm 2.7$ & $7.7 \pm 4.8$ & 0.670 & \\
\hline Glycerol & $3.0 \pm 2.5$ & $4.2 \pm 2.5$ & 0.877 & \\
\hline
\end{tabular}


creased by $9 \%$, whereas insulin levels increased by $25 \%$ $(5.9 \pm 0.1 \mathrm{mmol} / \mathrm{L}$ and $14.1 \pm 1.3 \mathrm{mU} / \mathrm{L}$ on average, respectively, at the end of the trial). Short-chain fructooligosaccharides did not affect fasting plasma glucose or insulin levels $(P=0.100$ and 0.204 for glucose and insulin, respectively; Table 1). The QUICKI, derived from the fasting plasma and insulin levels, did not improve by the supplementation of scFOS $(P=0.434$; Table 1). Fasting plasma triglyceride decreased by $20 \%$, whereas HDL cholesterol levels increased by $35 \%$ (0.109 \pm 0.005 and $0.741 \pm 0.054 \mathrm{mmol} / \mathrm{L}$, respectively, at the end of the trial). The triglyceride:HDL cholesterol ratio (fasting indicator of insulin resistance) did not improve by the supplementation of scFOS $(P=0.191$; Table 1). Urinary glucose excretion, which is a result of hyperglycemia (occurs when the threshold for glucose reabsorption is exceeded), was substantially higher $(P$ $<0.01$ ) in control and glucose calves compared with fructose and glycerol calves. Glucose excretion did not differ between scFOS and non-scFOS calves $(P=0.243$; Table 1).

In contrast with observations in other species (Respondek et al., 2008, 2011), scFOS supplementation did not improve insulin sensitivity in veal calves. It cannot be excluded that the absence of an effect of scFOS is related to the administered dose, which in this study was based on meta-analysis of the dose-response effect of scFOS supplementation on feed conversion ratio in veal calves (Kaufhold et al., 2000; Tai et al., 2009; Grand et al., 2013), because of the lack of data on the dose-response effect of scFOS supplementation on insulin sensitivity. We assumed that the feed conversion ratio is related to glucose utilization and thus might be related to insulin sensitivity. Based on BW, the administered dose in this trial was approximately 3 to 4 times lower than in previous studies in horses (in which scFOS improved insulin sensitivity; Respondek et al., 2011) and veal calves (in which scFOS improved glucose homeostasis; Kaufhold et al., 2000). Future studies need to focus on the dose-response effect of scFOS supplementation on insulin sensitivity in veal calves, if any. In some nonruminant species (e.g., dogs and horses), scFOS supplementation has been shown to improve insulin sensitivity. The absence of an effect of scFOS might therefore also be related to metabolic differences between ruminants and nonruminants. In calves, insulin sensitivity rapidly decreases during the first months of life (Stanley et al., 2002) independently of the weaning strategy (Stanley et al., 2002; Pantophlet et al., 2016b), and although differences in glucose homeostasis are observed as a result of dietary interventions, generally little to no effect on insulin sensitivity is found (Bach et al., 2013; Pantophlet et al., 2016a, c).
This might be related to the ontogenetic development of calves, as ontogenetic ruminants absorb little glucose from the intestinal tract and therefore may not have the genetic capacity to rapidly deal with large amounts of glucose. Consequently, absorbing large quantities of glucose from the MR disturbs glucose homeostasis in heavy veal calves. Mechanistic insight into species differences in the regulation of insulin sensitivity is required to obtain progress in this field. In conclusion, supplementation of scFOS to the MR did not improve insulin sensitivity in heavy veal calves.

\section{ACKNOWLEDGMENTS}

The authors thank Gerlof Reckman and Jeltje Kloosterman for their laboratory assistance (University Medical Centre Groningen). This project was jointly financed by the European Union, European Regional Development Fund and the Ministry of Economic Affairs, Agriculture and Innovation, Peaks in the Delta, the Municipality of Groningen, the Provinces of Groningen, Fryslân, and Drenthe as well as the Dutch Carbohydrate Competence Center (CCC2 WP21). Financial support was also provided by Tereos Syral, VanDrie Group, and Wageningen University.

\section{REFERENCES}

Bach, A., L. Domingo, C. Montoro, and M. Terre. 2013. Short communication: Insulin responsiveness is affected by the level of milk replacer offered to young calves. J. Dairy Sci. 96:4634-4637.

Grand, E., F. Respondek, C. Martineau, J. Detilleux, and G. Bertrand. 2013. Effects of short-chain fructooligosaccharides on growth performance of preruminant veal calves. J. Dairy Sci. 96:1094-1101.

Hostettler-Allen, R. L., L. Tappy, and J. W. Blum. 1994. Insulin resistance, hyperglycemia, and glucosuria in intensively milk-fed calves. J. Anim. Sci. 72:160-173.

Hugi, D., R. M. Bruckmaier, and J. W. Blum. 1997. Insulin resistance, hyperglycemia, glucosuria, and galactosuria in intensively milk-fed calves: Dependency on age and effects of high lactose intake. J. Anim. Sci. 75:469-482.

Kaufhold, J., H. M. Hammon, and J. W. Blum. 2000. Fructo-oligosaccharide supplementation: Effects on metabolic, endocrine and hematological traits in veal calves. J. Vet. Med. A Physiol. Pathol. Clin. Med. 47:17-29.

Muniyappa, R., S. Lee, H. Chen, and M. J. Quon. 2008. Current approaches for assessing insulin sensitivity and resistance in vivo: Advantages, limitations, and appropriate usage. Am. J. Physiol. Endocrinol. Metab. 294:E15-E26.

Pantophlet, A. J., W. J. J. Gerrits, R. J. Vonk, and J. J. G. C. van den Borne. 2016a. Substantial replacement of lactose with fat in a high-lactose milk replacer diet increases liver fat accumulation but does not affect insulin sensitivity in veal calves. J. Dairy Sci. 99:10022-10032.

Pantophlet, A. J., M. S. Gilbert, J. J. G. C. van den Borne, W. J. J. Gerrits, M. G. Priebe, and R. J. Vonk. 2016b. Insulin sensitivity in calves decreases substantially during the first 3 months of life and is unaffected by weaning or fructo-oligosaccharide supplementation. J. Dairy Sci. 99:7602-7611.

Pantophlet, A. J., M. S. Gilbert, J. J. G. C. van den Borne, W. J. J. Gerrits, H. Roelofsen, M. G. Priebe, and R. J. Vonk. 2016c. Lac- 
tose in milk replacer can partly be replaced by glucose, fructose or glycerol without affecting insulin sensitivity in veal calves. J. Dairy Sci. 99:3072-3080.

Respondek, F., K. Myers, T. L. Smith, A. Wagner, and R. J. Geor 2011. Dietary supplementation with short-chain fructo-oligosaccharides improves insulin sensitivity in obese horses. J. Anim. Sci. 89:77-83.

Respondek, F., K. S. Swanson, K. R. Belsito, B. M. Vester, A. Wagner, L. Istasse, and M. Diez. 2008. Short-chain fructooligosaccharides influence insulin sensitivity and gene expression of fat tissue in obese dogs. J. Nutr. 138:1712-1718.
Stanley, C. C., C. C. Williams, B. F. Jenny, J. M. Fernandez, H. G. Bateman, W. A. Nipper, J. C. Lovejoy, D. T. Gantt, and G. E. Goodier. 2002. Effects of feeding milk replacer once versus twice daily on glucose metabolism in Holstein and Jersey calves. J. Dairy Sci. 85:2335-2343.

Tai, X., X. Long, Z. Xiang, and F. Zuo. 2009. Effects of fructooligosaccharides on performance and blood biochemistry index and intestinal mucosa structure in early-weaned calves. Chin. J. Anim. Sci. 11:34-38. 